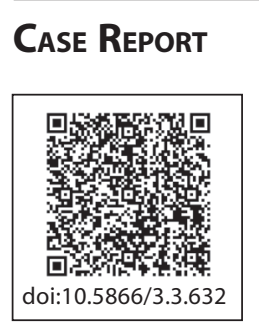

\title{
Rapidly progressive proliferativeverrucous leukoplakia - Case report \& Diagnostic difficulties
}

\author{
Praveen Kumar M11, Ajay Prakash P², Madhusudana Rao T³ ${ }^{3}$ Rashmi Santosh Kumar ${ }^{4}$
}

Department of Oral and maxillofacial Pathology,

Kamineni Institute of Dental Sciences,

Narketpalli, Nalgonda Dist., Andhra Pradesh, India.

Associate Professor ${ }^{1}$

Professor and H.O.D ${ }^{2}$

Senior Lecturer ${ }^{3}$

Associate Professor ${ }^{4}$

Article Info

Received: April 13, 2011

Review Completed: May, 17, 2011

Accepted: June, 18, 2011

Available Online: October, 2011

(c) NAD, 2011 - All rights reserved

\begin{abstract}
:
Proliferative verrucous leukoplakia (PVL) is a form of oral leukoplakia, which was first described in 1985 by Hansen et al. PVL is reported by various authors as very aggressive form of Leukoplakia due to its high rate of recurrence and a high rate of malignant transformation. Aetiology of PVL remains unclear as well as its diagnosis and management. In this article a case report and the criteria for diagnosis of Proliferative verrucous leukoplakia are discussed.
\end{abstract}

\section{Introduction:}

Oral leukoplakia (leuko $=$ white; plakia $=$ patch) is defined by the World Health Organization (WHO) as "a white patch or plaque that cannot be characterized clinically or pathologically as any other disease." The term is strictly a clinical one and does not imply a specific histopathologic tissue alteration. ${ }^{1}$

Proliferative verrucous leukoplakia (PVL) is a rare oral leukoplakia, characterized by proliferation, multiple foci of occurrences, and high rate of malignant transformation. ${ }^{2}$ Due to this since 1985 , PVL been segregated from other types of leukoplakia by Hansen. ${ }^{3}$ The aetiology of PVL is unclear. Unlike other forms of oral leukoplakia and oral squamous cell cancer, PVL lesions are not strongly associated with a history of alcohol or tobacco use or the presence of candidiasis, nor has evidence of immunodeficiency or vitamin deficiency. ${ }^{3}$

Viruses including human papillomavirus (HPV) ${ }^{4}$ and Epstein-Barr virus ${ }^{5}$ have been said to play a role in occurrence of PVL.

Email for correspondence:
Key words: Proliferative verrucous leukoplakia, Leukoplakia, oral, oral cancer.

\section{CASE REPORT:}

A 64 year old male patient visited private clinic, with a chief complaint of painless white patches on his left side of cheek inside the mouth since 7 months. Patient also gave history of slightly burning sensation on chewing of spicy foods since 10 days. Patient did not give any history of habit of tobacco usage both in chewing and smoking.

On Extra oral examination his face was symmetrical and nothing abnormal was detected. Intra oral Examination revealed an irregular shaped single white patch of approximately $2 \times 1 \mathrm{~cm}$. on the left side of the buccal mucosa. It was extending anteriorly from the distal margin of the lower left $2^{\text {nd }}$ premolar, posteriorly extending up to the mesial aspect of $2^{\text {nd }}$ molar (Fig.1). The surface of the lesion was almost smooth with few fissures in between. The surrounding mucosa was normal in structure and colour. On palpation, inspectory findings like site, shape, and extent were confirmed and the lesion was non scrapable. The lesion on palpation was non tender. It was provisionally diagnosed as smooth leukoplakia. 
A differential diagnosis of Verrucous carcinoma, Hypertrophic Candidiasis and plaque type Lichen Planus, was given. Incisional biopsy biopsy was made.

The $\mathrm{H}$ and $\mathrm{E}$ stained sections shows hyperkeratotic stratified squamous epithelium overlying a fibrous connective tissue. At one end epithelium was showing hyperplasia (Fig.2) and other slight atrophy. Histopathological diagnosis of Hyper ortho keratosis and epithelial atrophy was made. Patient was asked to visit the hospital for a regular check up once in a month.

On 3 months follow up a recurrent lesion on the same buccal mucosa and also a new lesion was seen on the right side of lower lip. The lesion on the buccal musosa was now showing more fissures on the surface. The surface of the lesion on the lower lip was exophytic with multiple nodular projections with fissures in between. They were provisionally diagnosed as verrucous carcinoma. Both the lesions were taken for incisional biopsy.

\section{Histo Pathology:}

The $\mathrm{H}$ and $\mathrm{E}$ stained sections of both the lesions showed mild dysplastic features like proliferative corrugated hyperkeratosis, Acanthosis, increased mitoses in basal and supra basal layers of epithelium. Rete processes are broad and regular and do not extended appreciably deeper than the general level of the basement membrane in the surrounding mucosa. The underlying fibrous connective tissue stroma shows mild chronic inflammatory cell infiltration (Fig.3).Finally by correlating with clinical features this case was diagnosed as a Proliferative Verrucous Leukoplakia.

\section{Discussion:}

PVL is a rare disease often seen in middle-aged and elderly women, occurring predominantly on the buccal mucosa, palate, gingiva, and tongue. Female to Male incidence ratio of occurrence is roughly $4: 1$. While women are most commonly affected on the buccal mucosa, men have lesions more commonly on the tongue. Only $30 \%$ of patients with proliferative verrucous leukoplakia give a history of smoking, ${ }^{3}$ whereas the smoking incidence is much higher in the patients affected by conventional leukoplakia. Aetiology of PVL is still enigma. Some authors suggested PVL might have an infectious etiology possibly a viral infection like HPV or EBV. Although several authors have suggested that HPV might have a role in the pathogenesis of $\mathrm{PVL}^{4}{ }^{4}$ Bagan et al. failed to find HPV in their group of patients and suggested that there is no association of PVL with $\mathrm{HPV}^{6}$ rather in their studies they have detected the presence of EBV in a large percent of patients. ${ }^{5}$

A number of the reported cases of PVL have initially presented as a solitary flat homogenous leukoplakia, while others present with multiple involved sites at time of diagnosis. Whatever be the initial presentation, recurrence after treatment is seen. Soon after first treatment the lesions appear again, not only at the previous site but also at new sites $^{7,8}$ - the gingiva being most commonly affected ${ }^{9}$ site. The patient reported here also showed initially a solitary lesion on the left buccal mucosa, and within a short duration the lesion appeared and also showed other site of involvement on the lip.

PVL exhibits persistent growth, eventually becoming exophytic and verrucous in nature ${ }^{1,11,12}$ As the lesions progress, they may transform into fullfledged squamous cell carcinoma (usually within 8 years of initial PVL diagnosis) 1,11,12 Hansen et al. described that PVL develops through a histopathological continuum encompassing 10 stages - from hyperkeratosis to squamous cell carcinoma. Later, Batsakis et al. proposed only 4 stages of continuum before forming Oral Squamous cell carcinoma. ${ }^{10}$ Histopathologically, PVL changes gradually from a simple plaque of hyperkeratosis without dysplasia to verrucous hyperplasia, followed by verrucous carcinoma finally Oral Squamous Cell Carcinoma.

Clinical differential diagnosis would range from Frictional Keratosis, Homogenous Leukoplakia, Papilloma, Papillary Hyperplasia, Cowdens Syndrome, Verrucous Hyperplasia and Verrucous Carcinoma.

The ambiguity of PVL is further aggravated because there are no criteria that dictate how extensive the leukoplakic changes should be or how many or which oral sub sites should be involved or how many recurrences should have occurred in order to qualify for the diagnosis of PVL. ${ }^{13}$ This lack of exact diagnostic criteria is a prime reason due to which patients of PVL do not get correct treatment.

The treatment that is recommended consists of multiple techniques such as $\mathrm{CO} 2$ laser surgery, 
surgery associated with radiotherapy, cryotherapy, retinoid $A$, systemic vitamin $A$ therapy, topical vitamins, bleomycin, and photodynamic therapy.

\section{CONCLUSION}

Proliferative verrucous leukoplakia is a rare but highly aggressive, Progressive form of oral leukoplakia. Patients with PVL are benefited if we have the earliest possible diagnosis and better treatment can be rendered to these patients, thus improving their prognosis. All the patients with a recurrent white lesion, however innocuous in appearance, should be suspected as PVL. Vigorous follow up is required. Continued biopsy of old and new lesions is a must. Have to be treated with aggressive approach. PVL can be diagnosed with confidence only in a retrospect and serial biopsy.

\section{References :}

1. Neville, Damm, Allen, Bouquot, Oral and maxillofacial pathology, 3rd edition, Saunders. pp 388- 397, 422- 423.

2. Hansen LS, Olson JA, Silverman S Jr. Proliferative verrucous leukoplakia. A long-term study of thirty patients. Oral Surg Oral Med Oral Pathol. 1985 Sep;60(3):285-98

3. Navarro CM, Sposto MR, Sgavioli-Massucato EM, Onofre MA.Transformation of proliferative verrucous leukoplakia to oral carcinoma: a ten years follow-up. Med Oral. 2004 May-Jul;9(3):229-33

4. Gopalakrishnan R, Weghorst CM, Lehman TA, Calvert RJ, Bijur G, Sabourin CL, et al. Mutated and wild-type p53 expression and HPV integration in proliferative verrucous leukoplakia and oral squamous cell carcinoma. Oral Surg Oral Med Oral Pathol Oral Radiol Endod. 1997;83:471-7.

5. Bagan JV, Jiménez Y, Murillo J, Poveda R, Díaz JM, Gavaldá $C$,et al. Epstein-Barr virus in oral proliferative verrucous leukoplakia and squamous cell carcinoma: A preliminary study. Med Oral Patol Oral Cir Bucal. 2008;13:E110-3.

6. Bagan JV, Jimenez Y, Murillo J, Gavaldá C, Poveda R, Scully $C$, et al. Lack of association between proliferative verrucous leukoplakia and human papillomavirus infection. J Oral Maxillofac Surg. 2007;65:46-9.

7. Silverman $S \mathrm{Jr}$, Gorsky M. Proliferative verrucous leukoplakia: a follow-up study of 54 cases. Oral Surg Oral Med Oral Pathol Oral Radiol Endod. 1997;84:154-7.

8. Fettig A, Pogrel MA, Silverman S Jr, Bramanti TE, Da Costa $M$, Regezi JA. Proliferative verrucous leukoplakia of the gingiva. Oral Surg Oral Med Oral Pathol Oral Radiol Endod. 2000;90:723-30.

9. Bagan JV, Jimenez $Y$, Sanchis JM, Poveda R, Milian MA, Murillo J, et al. Proliferative verrucous leukoplakia: high incidence of gingival squamous cell carcinoma. J Oral Pathol Med. 2003;32:379-82

10. Batsakis JG, Suarez P, El-Naggar AK. Proliferative verrucous leukoplakia and its related lesions. Oral Oncol. 1999;35:354-9.
11. Martin S. Greenberg, Michael Glick, Burket's Oral Medicine Diagnosis and Treatment 11th edn. BC Decker pp.88-89

12. Editorial, Oral proliferative verrucous leukoplakia revisited Oral Oncology,2008; 44: 719- 721.

13. Bishen KA, Sethi A. Proliferative Verrucous Leukoplakia diagnostic pitfalls and suggestions. Med Oral Patol Oral Cir Bucal. 2009.Jun 1;14 (6):E263-4.

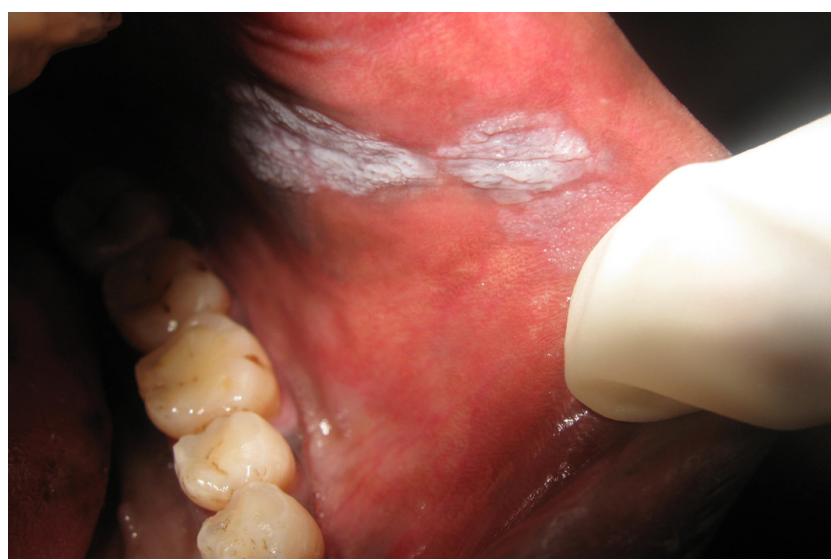

Fig.1. White patch (Leukoplakia) on the left buccal mucosa, showing few fissures.

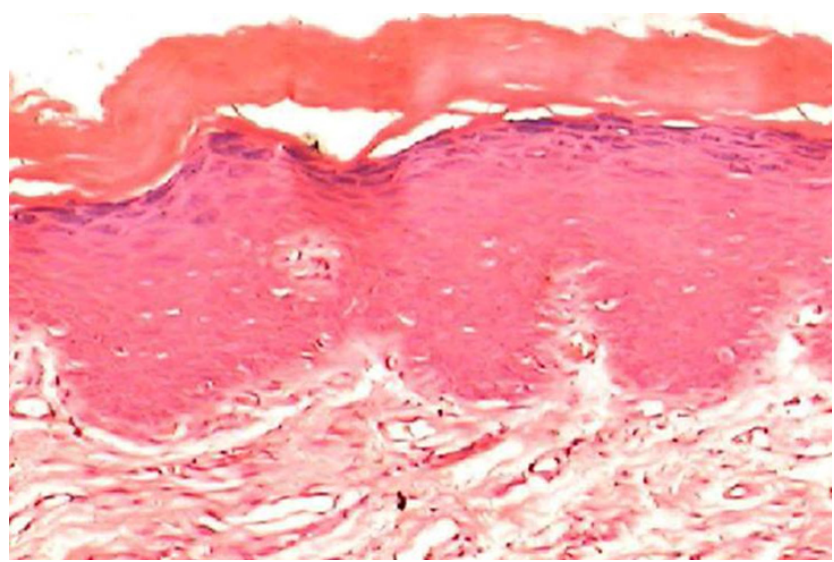

Fig.2. H \& E Section Showing Hyper Keratotic Epithelium

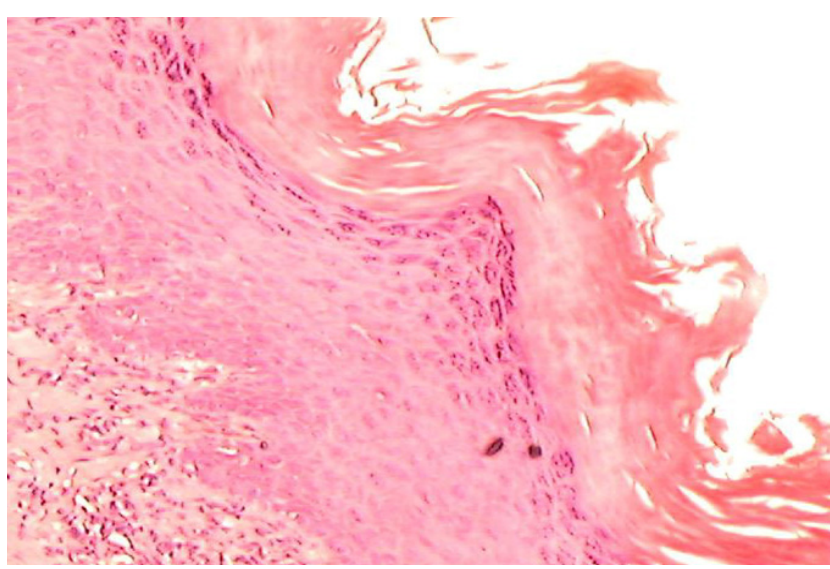

Fig.3. H\&E Section Showing Proliferative Corrugated Hyperkeratotic And Mild Dysplastic Epithelium 